justified if it improves outcome. NBS offers the potential for early intervention in order to preserve lung function and nutritional status. There are few, if any, ways of satisfactorily monitoring such babies non-invasively. To date the feasibility of recruiting newborn babies to invasive studies within the first few weeks following diagnosis is unknown.

Aim To assess the feasibility of recruiting and retaining infants diagnosed by NBS to an invasive observational study, prior to proposing an interventional study.

Methods All children presenting from the CF NBS programme from six centres comprising the London CF Collaboration (LCFC: Great Ormond St, Royal Brompton, Kings College, Royal London, Lewisham University and Epsom and St Helier's Hospitals) are being invited to participate in a study which involves infant lung function under sedation at $\sim 3$ and 12 months plus a combined CT, bronchoscopy and BAL under GA at $\sim 12$ months, within 3 weeks after the lung function test.

Results Since commencement of this study, 63 infants have screened positive for CF of whom five were ineligible (co-existent morbidities or moving away from area). Of the 58 eligible babies, seven have either declined (6) or withdrawn (1), eight families are currently considering participation and 43 have consented to participate. Infant lung function at 3 months has been undertaken in 43 babies (mean age at test 10.8 (SD 2.4) weeks. Of the 16 babies that have now reached one year of age, all have completed their follow-up lung function test and 15 have had their CT and BAL, with one awaiting an appointment. To date, parents' attitudes to taking part in this study have been universally positive.

Conclusions Despite initial concerns from some reviewers regarding the ability to recruit and retain families whose infants have been diagnosed with CF by NBS into clinical studies with invasive outcomes, these preliminary data indicate that it is possible to recruit a high proportion of babies to such a study. These results suggest that future interventional studies will be feasible.

\section{P73 THE COMPLEXITIES OF DEFINING ATOPY IN SEVERE CHILDHOOD ASTHMA}

doi:10.1136/thx.2010.150979.24

J Frith, L Fleming, C Bossley, N Ullmann, A Bush. Royal Brompton Hospital, London, UK

Background Defining atopy in children with severe therapy resistant asthma is complex. There is currently no gold standard test; skin prick testing (SPT) and allergen specific immunoglobulin E (sIgE) are both used. Atopy is increasingly considered to be a spectrum, not an all-or-none phenomenon (Allergy 2007;62:1379-86).

Hypothesis SPTs and sIgE cannot be used interchangeably, and that if both tests are not performed opportunities for intervention will be missed. Furthermore, severity of atopy will be defined differently by the two tests. Total IgE and fractional exhaled nitric oxide $\left(\mathrm{FeNO}_{50}\right)$ may also help quantify atopy.

Methods Cross-sectional study of 47 children with severe therapy resistant asthma, mean age 11.8 years (range $5.3-16.6$ years) who underwent measurement of a standard panel (house dust mite (HDM), dog, cat, weed pollens and grass pollens) of SPTs and SIgEs, total IgE and $\mathrm{FeNO}_{50}$ as part of their clinical work up.

Results Overall 42/47 (89\%) were atopic (defined as either a single positive SPT or SIgE). There was $98 \%$ concordance between the two tests in classifying atopy. When each allergen was considered individually, in 40/201 (19.8\%) the SPT and sIgE results were discordant (Abstract P73 Figure 1), most commonly, 25/201 (12.4\%) the SPT was negative and the SIgE was positive. HDM and cat sensitisation were more likely detected by sIgE, but dog by SPT. When atopy was quantified the sum of sIgEs compared with the sum of SPT wheal diameter showed only moderate correlation $(r=0.66, p=<0.001)$. Total IgE increased with an increasing number of positive sIgEs $(\mathrm{p}=0.028)$, but not significantly with number of positive SPTs There was a significant correlation between $\mathrm{FeNO}_{50}$ and total $\mathrm{IgE}$ $(\mathrm{r}=0.34, \mathrm{p}=0.02)$, but no difference in $\mathrm{FeNO}_{50}$ when patients were defined as atopic on either SPT or sIgE, and no relationship with increasing numbers of positive SPTs or sIgEs.

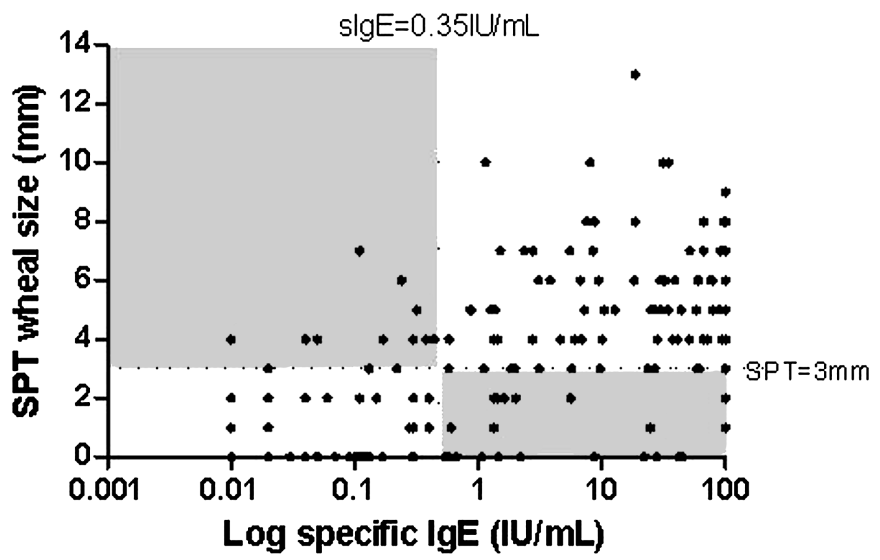

Abstract P73 Figure 1 Comparison of slgE and SPT wheal diameter for each individual allergen from the standard panel $(n=201)$. Dotted lines show the cut-off for a positive test result. Discordant results indicated by shaded areas.

Conclusions SPT and sIgE identify the group prevalence of atopy equally well; however for some allergens, concordance is poor, and when used to quantify atopy SPT and sIgE show only moderate correlation. If allergen avoidance is contemplated in children with severe therapy resistant asthma, then both tests should be performed in order to detect sensitisation.

\section{P74 BRITISH ASTHMA GUIDELINE REVISION DOES AFFECT PRESCRIBING PATTERNS IN CHILDREN}

doi:10.1136/thx.2010.150979.25

H Elkout, J S McLay, C R Simpson, P J Helms. Child Health, University of Aberdeen, Aberdeen, UK

Background Although audits of prescribing practice referenced to the British asthma management guidelines have been reported little is known on the impact of guideline revisions. Concerns about the use of high dose inhaled corticosteroids (ICS) in children, implicit in earlier adult oriented guidelines, has resulted in the promotion of add on therapy with long acting beta agonist (LABA) and/or leukotriene receptor antagonist (LTRA) and advice on age appropriate doses of short courses of oral corticosteroids (OCS) for exacerbations.

Methods Prescribing of asthma medication for children 0-18 years from 46 Scottish General Practices contributing to the Practice Team Information (PTI) database was assessed before (2001-2) and after the 2003 BTS/SIGN guideline revision ${ }^{1}$ (2005-6). PTI represents the rural/urban and socioeconomic make up of the whole Scottish population and includes 155230 children in the $0-18$ year age group (7.2-7.8\% with at least one prescription for an asthma medication per year).

Results In those children prescribed at least one asthma medication in each year of study, ICS at high dose $(>400 \mu \mathrm{g}$ beclometasone equivalent) decreased from $16.3 \%$ in $2001-2$ to $11.7 \%$ in $2005-6$ $(\mathrm{p}<0.001)$. This was accompanied by an increase in the prescribing of add-on therapy (LABA and/or LTRA) from $15.0 \%$ in $2001-2$ to $26.2 \%$ in $2005-6$. Prescribing of short courses (5-7 days) of OCS increased most prominently in children aged $<5$ years (from $6 \%$ in 